University of Wollongong

Research Online

Faculty of Engineering - Papers (Archive)

Faculty of Engineering and Information

Sciences

August 1991

\title{
Magnetic-field-induced indirect gap in a modulation-doped quantum well
}

D. M. Whittaker

Royal Signals and Radar Establishment, UK

T. A. Fisher

University of Wollongong

Philip E. Simmonds

University of Wollongong, simmonds@uow.edu.au

M. S. Skolnick

Royal Signals and Radar Establishment, UK

R. Smith

GEC Hirst Research Centre, UK

Follow this and additional works at: https://ro.uow.edu.au/engpapers

Part of the Engineering Commons

https://ro.uow.edu.au/engpapers/280

\section{Recommended Citation}

Whittaker, D. M.; Fisher, T. A.; Simmonds, Philip E.; Skolnick, M. S.; and Smith, R.: Magnetic-field-induced indirect gap in a modulation-doped quantum well 1991.

https://ro.uow.edu.au/engpapers/280

Research Online is the open access institutional repository for the University of Wollongong. For further information contact the UOW Library: research-pubs@uow.edu.au 


\title{
Magnetic-Field-Induced Indirect Gap in a Modulation-Doped Quantum Well
}

\author{
D. M. Whittaker, ${ }^{(1)}$ T. A. Fisher, ${ }^{(2)}$ P. E. Simmonds, ${ }^{(2)}$ M. S. Skolnick, ${ }^{(1),(a)}$ and R. S. Smith ${ }^{(3)}$ \\ ${ }^{(1)}$ Royal Signals and Radar Establishment, St. Andrews Road, Malvern, Worcestershire, WRI 4 3PS, United Kingdom \\ ${ }^{(2)}$ Department of Physics, University of Wollongong, Wollongong, New South Wales 2500, Australia \\ ${ }^{(3)}$ GEC Hirst Research Centre, Wembley, Middlesex, HA9 7PP, United Kingdom
}

(Received 21 February 1991)

\begin{abstract}
We report the first experimental evidence for the indirect fundamental band gap developed when an in-plane magnetic field is applied to a modulation-doped quantum well. The observed photoluminescence peak for the lowest subband undergoes a large, approximately quadratic shift with field, a consequence of the behavior of the allowed transitions in the increasingly indirect-gap band structure. A good theoretical fit is obtained by calculating the transition energies using a realistic self-consistent Hartree potential for the quantum-well profile.
\end{abstract}

PACS numbers: 78.20.Ls, 73.20.Dx, 75.20.Ck, 78.55.Cr

The behavior of the two-dimensional electron gas in a modulation-doped quantum well with an in-plane magnetic field is a consequence of the interplay of electrostatic, confinement, and magnetic forces. The eigenstates evolve from bound states of the confining potential at zero field to Landau levels at high fields, their character being determined by the relative magnitudes of the cyclotron radius, $a_{H}=(\hbar / e B)^{1 / 2}$, and the width of the potential, $d$ [1]. In the low-field regime $\left(a_{H}>d\right)$, the energy of the bottom of the subband undergoes a diamagnetic shift determined by the extent of the wave function. At higher fields, as the magnetic confinement dominates $\left(a_{H}<d\right)$, each subband develops some of the characteristics of a Landau level: The energy tends asymptotically towards a linear shift with field, and there is an increase in the effective mass, and hence density of states. This type of behavior has been observed in quantum wells and accumulation layers, in transport measurements [2,3], in intersubband magnetoabsorption [4,5], and in photoluminescence (PL) [6].

The presence of the in-plane field may also change the nature of the subband structure, due to the relationship between $k_{x}$, the in-plane momentum perpendicular to the field $(B \| y)$, and the position of the orbit center on the growth axis, $z_{0}=a_{H}^{2} k_{x}$. The energy of a state depends on the location of its orbit center in the structure, so the $k_{x}$ dispersion is determined by the details of the potential. In particular, the minimum of a subband will occur at $k_{x}$ corresponding to an orbit centered where the state is localized [7]. Thus in a modulation-doped structure, if electrostatic effects are strong enough to separate the electron and hole states, the in-plane magnetic field will cause an indirect band gap. The $k$-space gap $\Delta k$ will be proportional to the spatial separation $\Delta z$ of the electron and hole and to the magnetic field through $\Delta k=\Delta z / a_{H}^{2}$. Such distortions of the dispersion have been widely discussed theoretically [8-11], but the only experimental evidence is in the form of additional transitions in intersubband magnetoabsorption measurements [5], which do not probe the nature of the conduction-valence-band gap.

In this Letter, we present PL measurements on a 250 -
$\AA$, asymmetrically doped quantum well which give the first experimental verification of a magnetic-field-induced indirect fundamental band gap. Our results show that the lowest, $n=1$, electron-hole recombination peak energy exhibits a large, quadratic increase with field $(0.19$ $\mathrm{meV} / \mathrm{T}^{2}$ ), an order of magnitude greater than that expected for the shift of the band extrema. Since the gap is indirect in $k$ space, the allowed transitions involving holes thermalized to the top of the valence band correspond to electron states above the bottom of the conduction subbands. The energy shift of the transition gives a measure of the $k$-space separation, according to $\Delta E \sim \hbar^{2} \Delta k^{2} /$ $2 m_{e}$, if the small diamagnetic shift of the bottom of the subbands is neglected. Since $\Delta k$ is proportional to the field, this leads to the large quadratic variation with field observed experimentally. Similar behavior has in fact been reported by some of the present authors in very wide (700 ̊) InGaAs-InP quantum wells [12], but without interpretation at the time.

The experiments were carried out at $2 \mathrm{~K}$, with the PL excited by $0.5 \mathrm{~W} \mathrm{~cm}^{-2}$ of radiation from a He- $\mathrm{Ne}$ laser. The PL was dispersed by a $0.75-\mathrm{m}$ grating spectrometer and detected with a cooled Ge photodiode. The results discussed here were from a $250-\AA \operatorname{In}_{0.11} \mathrm{Ga}_{0.89}$ As quantum well, with a thick barrier of GaAs on the substrate side and $350 \AA$ of $\mathrm{Al}_{0.23} \mathrm{Ga}_{0.77} \mathrm{As}$ on the other. The AlGaAs is heavily $n$ doped $\left(n=1.5 \times 10^{18} \mathrm{~cm}^{-3}\right)$, except for a spacer layer of $100 \AA$ immediately adjacent to the well. This structure is particularly suited to the investigation of the indirect gap because the asymmetry and high doping lead to strong electric fields and the wide well allows a large separation of electrons and holes. Further studies on other samples with the same general design, but well widths of 150 and $200 \AA$, gave qualitatively very similar results, though the shift rates were reduced due to the smaller spatial separations of the electrons and holes.

The zero-field behavior of our structures has been investigated theoretically [13] by solving self-consistently the Schrödinger and Poisson equations. The resulting potential in the region of the well is shown in Fig. 1, along with the wave functions of the hole and two occupied 


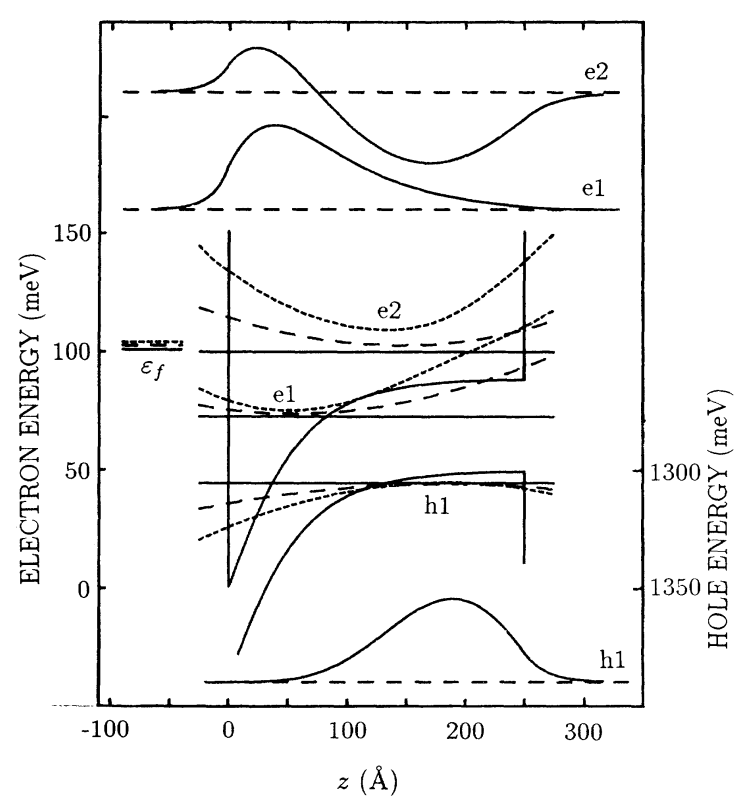

FIG. 1. Zero-field self-consistent potential in the region of the well, and wave functions for the hole and occupied electron subbands. The origin of energy is the conduction-band edge at the extreme left-hand side of the well. The curves superimposed on the potential are the carrier dispersions, plotted as a function of orbit center $z_{0}$ (see text). The solid lines correspond to zero field, long-dashed lines to $7 \mathrm{~T}$, and short-dashed lines to $12 \mathrm{~T}$.

electron subbands. The transfer of carriers from the donors in the barrier to lower-energy states in the well produces strong local electric fields and thus considerable band bending. As a result, the lowest-energy $n=1$ electron and hole subbands are localized on oppsite sides of the well, with the second electron subband $27 \mathrm{meV}$ higher in energy and extended over the whole well. The Fermi energy is calculated to be about $1 \mathrm{meV}$ above the bottom of the second subband, giving a total sheet charge density of $8.4 \times 10^{11} \mathrm{~cm}^{-2}$, in good agreement with Shubnikov-de Haas measurements which indicate a value of $9 \times 10^{11} \mathrm{~cm}^{-2}$.

These theoretical results account well for the zero-field photoluminescence spectrum in Fig. 2. The peaks $E_{11}$ and $E_{21}$, corresponding to recombination of holes in the $n=1$ subband with $n=1$ and 2 electrons, respectively, have a separation of $27 \mathrm{meV}$. The actual Fermi energy, obtained from PL measurements with $B \|_{z}$, is about 3 meV above the bottom of the second subband. Despite the much smaller population the $E_{21}$ peak is $\sim 20$ times stronger than $E_{11}$. This principally reflects the large difference in overlap of electron and hole wave functions for the two subbands, evident in Fig. 1, which causes both matrix element and excitonic effects to favor the higherenergy peak [13]. The $E_{11}$ peak is asymmetrically broadened to higher energy by the "forbidden" $\delta k \neq 0$ transitions involving electrons further up in the subband,

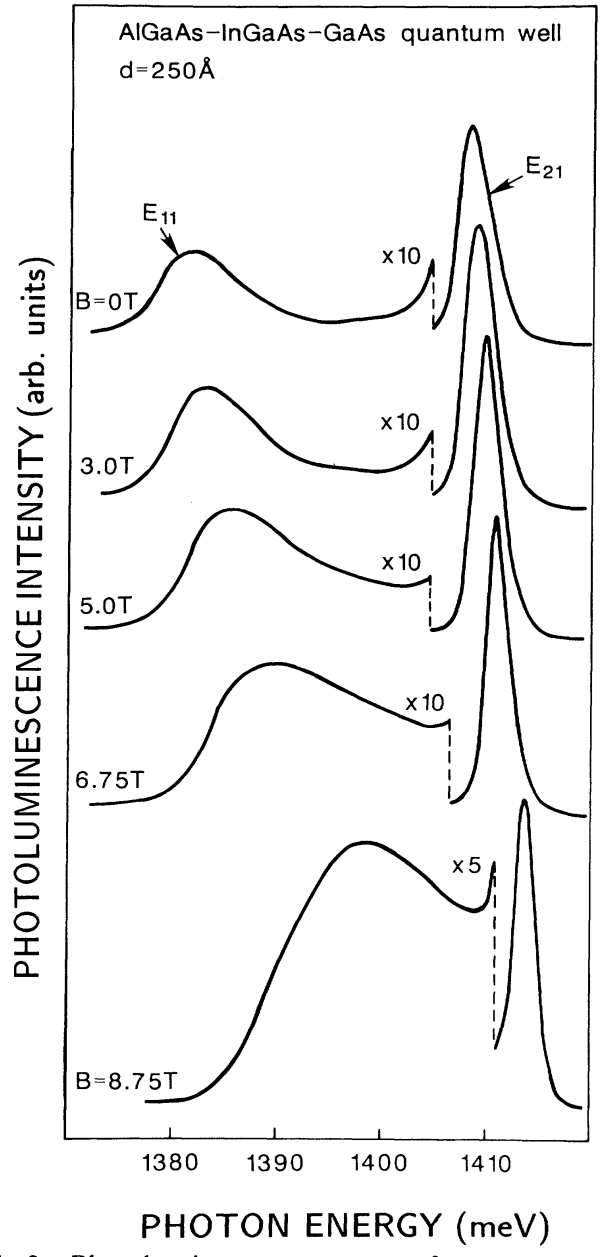

FIG. 2. Photoluminescence spectra for a range of in-plane magnetic fields. The peaks $E_{11}$ and $E_{21}$ correspond to allowed transitions from the $n=1$ and 2 electron subbands.

made weakly allowed by disorder or a thermal population of holes away from the valence-band maximum. Extrapolation of the Landau-level energies in measurements with $B \| z$ shows that the $E_{11}$ peak occurs at the bottom of the subband, indicating that the disorder is weak, so the peak energies coincide with the allowed transitions. Figure 2 also shows the PL spectra for a range of in-plane magnetic fields. The $E_{11}$ peak shows the anomalously large quadratic upshift with field, indicative of the indirect nature of the gap. Magnetic depopulation of the $n=2$ subband only becomes apparent at the highest field shown $(8.75 \mathrm{~T})$, a consequence of the relatively weak magnetic effects on the density of states and the small differential diamagnetic shift of the two occupied subbands.

The increase of peak energies with field is shown by the experimental points in Fig. 3. The quadratic behavior is very apparent, with shift rates of $0.19 \pm 0.02$ and 0.07 $\mathrm{meV} / \mathrm{T}^{2}$ for $n=1$ and 2 transitions, respectively. These 


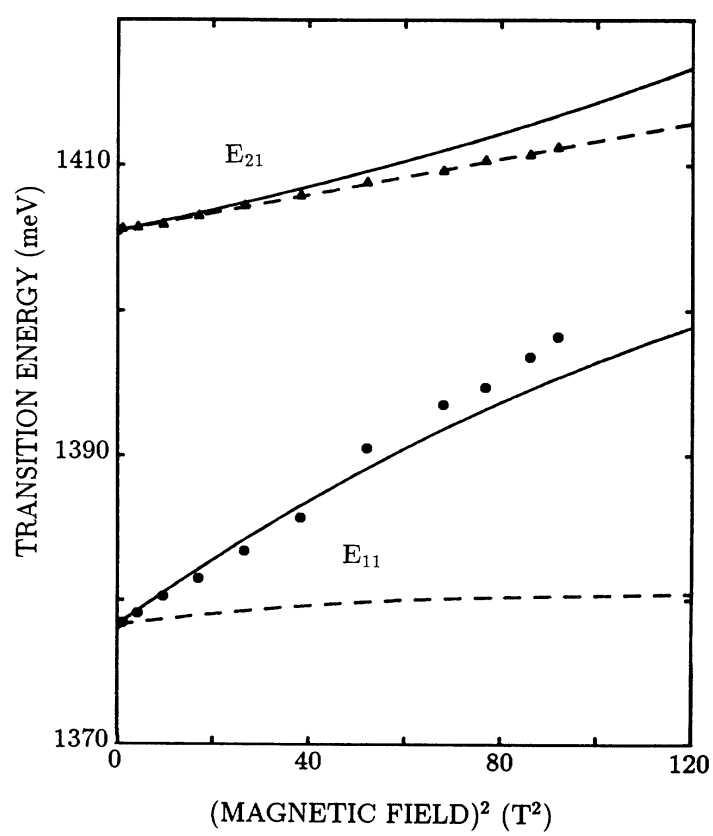

FIG. 3. Experimental (points) and theoretical (solid lines) transition energies as a function of the square of the field. Also shown (dashed lines) are the diamagnetic shifts of the bottoms of the subbands.

compare with values of 0.02 and $0.07 \mathrm{meV} / \mathrm{T}^{2}$ calculated for the diamagnetic shifts of the bottom of the subbands (dashed lines). The observed $n=1$ transition clearly cannot be between the band extrema, since the shift rate is much larger than the calculated value, and is significantly greater than that for the $n=2$ peak. It is also an order of magnitude larger than the value $\left(0.018 \mathrm{meV} / \mathrm{T}^{2}\right)$ obtained in the PL experiments of Ref. [6], involving a narrower $(150 \AA)$ symmetric well, which remains a direct gap since the electrostatic forces are insufficient to separate the electrons and holes. However, allowing for the indirect gap, even the simple result above $\left(\Delta E=\hbar^{2} \Delta z^{2} / 2 m_{e} a_{H}^{4}\right)$ gives fair agreement for the transition energies: The shift rate of $0.19 \mathrm{meV} / \mathrm{T}^{2}$ corresponds to a spatial separation $\Delta z$ of approximately $120 \AA$, very reasonable for the wave functions in Fig. 1 .

In order to put the theory on a more accurate footing, it is necessary to include the magnetic field with the electrostatic well potential in the self-consistent solution. This problem has been considered by a number of authors [1,8-11], with various degrees of sophistication in the treatment of the potential, band structure [10], selfconsistency [8-11], and more complicated many-body effects [9]. The approach adopted here is to treat electron-electron interactions only in the Hartree approximation, but to include the modification of the dispersion and wave functions by the magnetic field in the selfconsistency loop. In fact, the effects of the relatively low fields used here in the self-consistency procedure turn out

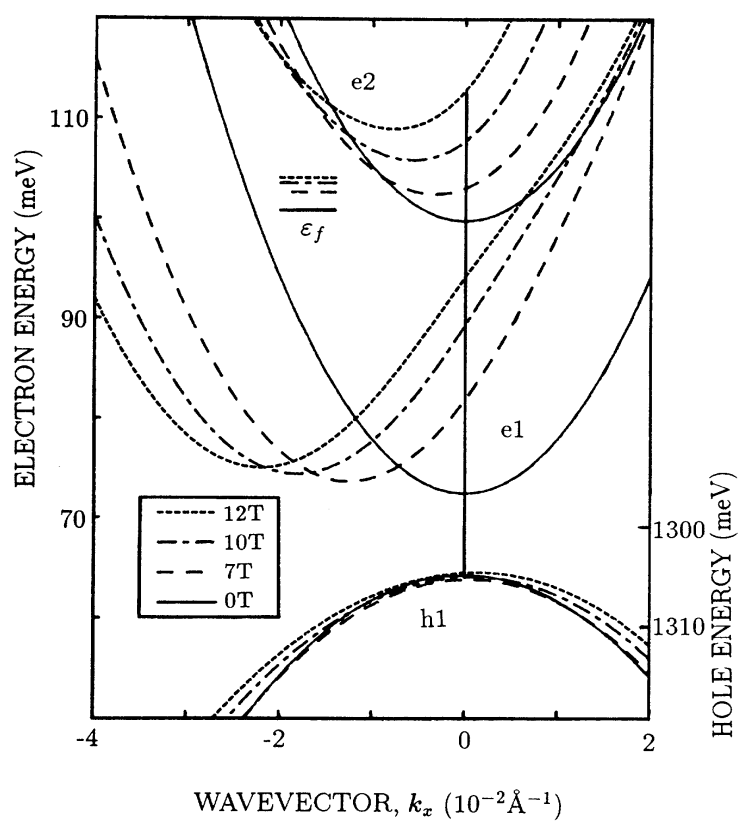

FIG. 4. Theoretical dispersions for the occupied subands at various fields, plotted as a function of $k_{x}$. The origin of energy is chosen as in Fig. 1. Allowed transitions for a thermalized hole are indicated by the vertical solid line.

to be of little importance: The Hartree potential varies only slightly with field, and the calculated transition energies are changed by $<1 \mathrm{meV}$.

In solving the Schrödinger equation, the conductionband offset at the edges of the wells is taken to be $70 \%$ of the total discontinuity. Since the In content is small, the InGaAs carrier masses are assumed to be the same as in GaAs. The in-plane hole dispersion is treated as parabolic, with mass $0.15 m_{e}$. Although this is unrealistic, the hole diamagnetism contributes $<10 \%$ of the total upshift, so even sizable errors can be tolerated.

In Fig. 1 the calculated electron and hole dispersions plotted as a function of orbit center $z_{0}$ are superimposed on the zero-field potential. By using $z_{0}$ instead of $k_{x}$, the relationship between the indirect gap and the form of the potential is made clear. Although the orbit center separation $\Delta z_{0}$ of the extrema remains roughly constant, as determined by the potential, the $k$-space gap increases in proportion to the field, since $\Delta z_{0}=a_{H}^{2} \Delta k$. This may be seen in Fig. 4 where the calculated band structure is plotted in the more usual way as a function of $k_{x}$. The zero of $z$, which determines the point $k_{x}=0$, is chosen to be the low-field maximum of the hole dispersion. Since this point shifts very little in $z$ with field $(<5 \AA)$, its shift in $k$ space is also small.

The $k$-space gaps in Fig. 4 appear to be much larger than those calculated by Oliveira et al. [11] for similar structures. However, the allowed transitions with their sign conventions have $k_{e}=-k_{h}$, so to make a direct com- 
parison it is necessary to reflect one of the bands in Fig. 11 of Ref. [11] around $k=0$, upon which the gaps become similar in magnitude to those calculated here. One consequence of this is that, for asymmetric wells, the effects of the indirect gap are much greater than is suggested in Ref. [11], as is demonstrated by the present results.

In Fig. 3, the experimental peak energies are compared with the calculated direct transitions (solid lines) [14]. Also shown (dashed lines) is the behavior of the indirect gaps, corresponding to the change in separation of the subband extrema with field. The agreement for the $n=1$ transition with the solid curve is very good, and clearly shows that the fundamental band gap is indeed indirect. The situation for the $n=2$ transition is less clear, as it seems to follow closely the indirect gap, though the difference between direct and indirect transitions is much smaller than for $n=1(\sim 2 \mathrm{meV}$ at 9.6 T). However, such behavior is not unreasonable, since at the higher fields, where the difference becomes significant, the $n=2$ subband is depopulating. When few electrons remain with sufficient $k$ to recombine in a direct transition, the peak must move to lower energy.

To conclude, by measuring the shift rate with field of the allowed photoluminescence transitions, we have demonstrated that an in-plane magnetic field produces an indirect band gap in an asymmetric modulation-doped quantum well. This verifies previous theoretical predictions. The experimental results are in good quantitative agreement with self-consistent calculations for the struc- ture considered.

(a) Present address: Department of Physics, University of Sheffield, Sheffield, S3 7RH, United Kingdom.

[1] F. Stern, Phys. Rev. Lett. 21, 1687 (1968).

[2] D. C. Tsui, Solid State Commun. 9, 1789 (1971).

[3] R. E. Doezema, M. Nealon, and S. Whitmore, Phys. Rev. Lett. 45, 1593 (1980).

[4] W. Beinvogl, A. Kamgar, and J. F. Koch, Phys. Rev. B 14, 4274 (1976).

[5] S. Oelting, U. Merkt, and J. P. Kotthaus, Surf. Sci. 170, 402 (1986).

[6] A. J. Turberfield, J. F. Ryan, and J. M. Worlock, Surf. Sci. 170, 624 (1986).

[7] The low-field dispersion minimum will occur at $k_{x}$ corresponding to the $z_{0}$ which minimizes the expectation value of the diamagnetic term $\left(z-z_{0}\right)^{2}$.

[8] T. Ando, J. Phys. Soc. Jpn. 39, 411 (1975).

[9] T. Ando, J. Phys. Soc. Jpn. 44, 475 (1978).

[10] W. Zawadzki, S. Klahn, and U. Merkt, Phys. Rev. B 33, 6916 (1986).

[11] G. M. G. Oliveira, V. M. S. Gomes, A. S. Chaves, J. R. Leite, and J. M. Worlock, Phys. Rev. B 35, 2896 (1987).

[12] P. E. Simmonds, M. S. Skolnick, L. L. Taylor, S. J. Bass, and K. J. Nash, Solid State Commun. 67, 1151 (1988).

[13] M. S. Skolnick, D. M. Whittaker, P. E. Simmonds, T. A. Fisher, M. K. Saker, J. M. Rorison, R. S. Smith, P. B. Kirby, and C. R. H. White, Phys. Rev. B 43, 7354 (1991).

[14] A small energy shift has been added to the theoretical results to ensure that the $n=1$ transition at zero field lines up exactly with the experimental value. 